\title{
Fermionic vacuum densities in higher-dimensional de Sitter spacetime
}

\author{
E. R. Bezerra de Mello ${ }^{1 *}$ and A. A. Saharian ${ }^{1,2 \dagger}$ \\ ${ }^{1}$ Departamento de Física-CCEN, Universidade Federal da Paraíba \\ 58.059-970, Caixa Postal 5.008, João Pessoa, PB, Brazil \\ ${ }^{2}$ Department of Physics, Yerevan State University, \\ 1 Alex Manoogian Street, 0025 Yerevan, Armenia
}

February 14, 2019

\begin{abstract}
Fermionic condensate and the vacuum expectation values of the energy-momentum tensor are investigated for twisted and untwisted massive spinor fields in higher-dimensional de Sitter spacetime with toroidally compactified spatial dimensions. The expectation values are presented in the form of the sum of corresponding quantities in the uncompactified de Sitter spacetime and the parts induced by non-trivial topology. The latter are finite and the renormalization is needed for the first parts only. Closed formulae are derived for the renormalized fermionic vacuum densities in uncompactified odd-dimensional de Sitter spacetimes. It is shown that, unlike to the case of 4-dimensional spacetime, for large values of the mass, these densities are suppressed exponentially. Asymptotic behavior of the topological parts in the expectation values is investigated in the early and late stages of the cosmological expansion. When the comoving lengths of compactified dimensions are much smaller than the de Sitter curvature radius, to the leading order the topological parts coincide with the corresponding quantities for a massless fermionic field and are conformally related to the corresponding flat spacetime results. In this limit the topological parts dominate the uncompactified de Sitter part and the back-reaction effects should be taken into account. In the opposite limit, for a massive field the asymptotic behavior of the topological parts is damping oscillatory.
\end{abstract}

PACS numbers: 04.62.+v, 04.50.-h, 11.10.Kk, 04.20.Gz

\section{Introduction}

De Sitter (dS) spacetime is one of the simplest and most interesting spacetimes allowed by general relativity. Quantum field theory in this background has been extensively studied during the past two decades. Much of early interest to dS spacetime was motivated by the questions related to the quantization of fields propagating on curved backgrounds. This spacetime has a high degree of symmetry and numerous physical problems are exactly solvable on this background. The

\footnotetext{
*E-mail: emello@fisica.ufpb.br

${ }^{\dagger}$ E-mail: saharian@ictp.it
} 
importance of this theoretical work increased by the appearance of the inflationary cosmology scenario [1. In most inflationary models an approximately dS spacetime is employed to solve a number of problems in standard cosmology. During an inflationary epoch quantum fluctuations in the inflaton field introduce inhomogeneities and may affect the transition toward the true vacuum. These fluctuations play a central role in the generation of cosmic structures from inflation. More recently astronomical observations of high redshift supernovae, galaxy clusters and cosmic microwave background [2] indicate that at the present epoch the Universe is accelerating and can be well approximated by a world with a positive cosmological constant. If the Universe would accelerate indefinitely, the standard cosmology would lead to an asymptotic dS universe. Hence, the investigation of physical effects in dS spacetime is important for understanding both the early Universe and its future.

Many of high energy theories of fundamental physics are formulated in higher-dimensional spacetimes. In particular, the idea of extra dimensions has been extensively used in supergravity and superstring theories. It is commonly assumed that the extra dimensions are compactified. From an inflationary point of view universes with compact spatial dimensions, under certain conditions, should be considered a rule rather than an exception [3]. The models of a compact universe with non-trivial topology may play an important role by providing proper initial conditions for inflation. As it was argued in Refs. 44, there is no reason to believe that the version of dS spacetime which may emerge from string theory, will necessarily be the most familiar version with symmetry group $O(1,4)$ and there are many different topological spaces which can accept the dS metric locally. There are many reasons to expect that in string theory the most natural topology for the universe is that of a flat compact three-manifold. The quantum creation of the universe having toroidal spatial topology is discussed in [5] and in references [6] within the framework of various supergravity theories.

The compactification of spatial dimensions leads to a number of interesting quantum field theoretical effects which include instabilities in interacting field theories [7], topological mass generation [8], symmetry breaking [9]. In the case of non-trivial topology the boundary conditions imposed on fields give rise to the modification of the spectrum for vacuum fluctuations and, as a result, to the Casimir-type contributions in the vacuum expectation values of physical observables (for the topological Casimir effect and its role in cosmology see [10, 11] and references therein). In the Kaluza-Klein-type models the Casimir effect has been used as a stabilization mechanism for moduli fields which parametrize the size and the shape of the extra dimensions. The Casimir energy can also serve as a model for dark energy needed for the explanation of the present accelerated expansion of the universe (see [12] and references therein). One-loop quantum effects for various spin fields on the background of dS spacetime have been discussed by several authors (see, for instance, [13-28] and references therein). The effects of the toroidal compactification of spatial dimensions in dS spacetime on the properties of quantum vacuum for a scalar field with general curvature coupling parameter are investigated in Refs. [29, 30] (for quantum effects in braneworld models with dS spaces and in higher-dimensional brane models with compact internal spaces see, for instance, Refs. [31, 32]). The one-loop quantum effects for a fermionic field on background of 4-dimensional dS spacetime with spatial topology $\mathrm{R}^{p} \times\left(\mathrm{S}^{1}\right)^{q}$ are studied in 33 .

In the present paper, we investigate one-loop quantum effects arising from vacuum fluctuations of a fermionic field on background of higher-dimensional dS spacetime with toroidally compactified spatial dimensions. The important quantities that characterize the quantum fluctuations during the dS expansion are the fermionic condensate and the expectation value of the energy-momentum tensor. In the next section, by using the dimensional regularization procedure, we evaluate these quantities in uncompactified odd-dimensional dS spacetimes. The plane wave fermionic eigenfunctions in $(D+1)$-dimensional dS spacetime with an arbitrary number 
of toroidally compactified dimensions are constructed in section 3 . In section 4 these eigenfunctions are used for the evaluation of the fermionic condensate in both cases of the fields with periodicity and antiperiodicity conditions along compactified dimensions. The behavior of these quantities is investigated in asymptotic regions of the parameters. The topological parts in the vacuum expectation values of the energy-momentum tensor are investigated in section 5. In the last section we summarize the main results of the paper.

\section{Vacuum expectation values in odd-dimensional uncompactified dS spacetime}

In this section we will evaluate the fermionic condensate and the vacuum expectation value $(\mathrm{VEV})$ of the energy-momentum tensor in odd-dimensional uncompactified dS spacetimes by using the dimensional regularization procedure. These quantities are among the most important characteristics of the vacuum state and carry an information on both local and global properties. Consider a quantum fermionic field $\psi$ on background of $(D+1)$-dimensional de Sitter spacetime, $\mathrm{dS}_{D+1}$, described by the line element

$$
d s^{2}=d t^{2}-e^{2 t / \alpha} \sum_{i=1}^{D}\left(d z^{i}\right)^{2}
$$

where the parameter $\alpha$ in the expression for the scale factor is related to the corresponding cosmological constant $\Lambda$ by the formula $\alpha^{2}=D(D-1) /(2 \Lambda)$.

The dynamics of the field in a curved spacetime is governed by the covariant Dirac equation

$$
i \gamma^{\mu} \nabla_{\mu} \psi-m \psi=0, \nabla_{\mu}=\partial_{\mu}+\Gamma_{\mu}
$$

where $\gamma^{\mu}=e_{(a)}^{\mu} \gamma^{(a)}$ are the generalized Dirac matrices and $\Gamma_{\mu}$ is the spin connection. The latter is given in terms of the flat-space Dirac matrices $\gamma^{(a)}$ by the relation

$$
\Gamma_{\mu}=\frac{1}{4} \gamma^{(a)} \gamma^{(b)} e_{(a)}^{\nu} e_{(b) \nu ; \mu},
$$

where the semicolon means the covariant derivative of vector fields. In the equations above $e_{(a)}^{\mu}$ are the tetrad components defined by $e_{(a)}^{\mu} e_{(b)}^{\nu} \eta^{a b}=g^{\mu \nu}$, with $\eta^{a b}$ being the Minkowski spacetime metric tensor.

Let $S_{F}\left(x, x^{\prime}\right)$ be the Feynman Green function for the spinor field in $(D+1)$-dimensional dS spacetime. In Ref. [14] it has been shown that for $D<1$ one has the relation

$$
\operatorname{tr}\left[S_{F}(x, x)\right]=-\frac{i N m}{(4 \pi)^{(D+1) / 2}} \frac{\Gamma\left(\frac{D+1}{2}+i m \alpha\right) \Gamma\left(\frac{D+1}{2}-i m \alpha\right)}{\alpha^{D-1} \Gamma(1-i m \alpha) \Gamma(1+i m \alpha)} \Gamma\left(\frac{1-D}{2}\right),
$$

where the trace is taken over the spinor indices of the Green function and $N$ is the number of spinor components in $(D+1)$-dimensional spacetime (see next section). The expression on the right-hand side of this formula is finite for all even values of $D$. In this case, by using the well-known properties of the gamma function, formula (4) can also be written in the form

$$
\operatorname{tr}\left[S_{F}(x, x)\right]=-\frac{i N m^{D}}{(4 \pi)^{(D+1) / 2}} \Gamma\left(\frac{1-D}{2}\right) \tanh (\pi m \alpha) \prod_{j=0}^{D / 2-1}\left[1+\frac{(j+1 / 2)^{2}}{m^{2} \alpha^{2}}\right] .
$$


To find the renormalized value for the fermionic condensate we should subtract from (5) the corresponding DeWitt-Schwinger expansion $\operatorname{tr}\left[S_{F}^{(\mathrm{DS})}(x, x)\right]$, truncating the expansion at the adiabatic order $D$ :

$$
\langle\bar{\psi} \psi\rangle_{\mathrm{dS}, \mathrm{ren}}=-i\left\{\operatorname{tr}\left[S_{F}(x, x)\right]-\operatorname{tr}\left[{ }^{(D)} S_{F}^{(\mathrm{DS})}(x, x)\right]\right\}
$$

where ${ }^{(D)} S_{F}^{(\mathrm{DS})}(x, x)$ stands for the truncated expansion. This expansion can be found by using the corresponding result for the bispinor $G_{F}\left(x, x^{\prime}\right)$ which is related to the function $S_{F}\left(x, x^{\prime}\right)$ by the formula $S_{F}\left(x, x^{\prime}\right)=\left(i \gamma^{\mu} \nabla_{\mu}+m\right) G_{F}\left(x, x^{\prime}\right)$. The structure of the DeWitt-Schwinger expansion for the function $G_{F}\left(x, x^{\prime}\right)$ is similar to that for the scalar Green function given, for example, in [28]:

$$
G_{F}^{(\mathrm{DS})}(x, x)=\frac{-i m^{D-1}}{(4 \pi)^{(D+1) / 2}} \sum_{j=0}^{\infty} \frac{a_{j}(x)}{m^{2 j}} \Gamma(j-(D-1) / 2),
$$

where the coefficients $a_{j}(x)$ are bispinors. The expressions for the first three coefficients in terms of the curvature tensor of the background geometry can be found in [34. In particular, $a_{0}=I$, $a_{1}=-R I / 12$, where $I$ is the unit matrix and $R$ is the Ricci scalar. For de Sitter spacetime the bispinor coefficients $a_{j}(x)$ in the adiabatic expansion of $G_{F}\left(x, x^{\prime}\right)$ have the structure $a_{j}(x)=$ $b_{j} R^{j} I$, where $b_{j}$ are numerical coefficients and $R=D(D+1) / \alpha^{2}$ is the Ricci scalar for $\mathrm{dS}_{D+1}$. For example, the coefficient $a_{2}$ is given by the expression

$$
a_{2}=\frac{D(D+1)}{1440 \alpha^{4}}(D-2)(5 D+7) I .
$$

Now, by using the relation $\operatorname{tr}\left[\gamma^{\mu}\right]=0$ for the Dirac matrices, we see that for dS spacetime one has the relation $\operatorname{tr}\left[S_{F}^{(\mathrm{DS})}(x, x)\right]=m \operatorname{tr}\left[G_{F}^{(\mathrm{DS})}(x, x)\right]$. Hence, by making use of formulae (6) and (7), for the renormalized value of the fermionic condensate we find:

$$
\begin{aligned}
\langle\bar{\psi} \psi\rangle_{\mathrm{dS}, \text { ren }}= & -\frac{N m^{D}}{(4 \pi)^{(D+1) / 2}} \Gamma\left(\frac{1-D}{2}\right)\left\{\tanh (\pi m \alpha) \prod_{j=0}^{D / 2-1}\left[1+\frac{(j+1 / 2)^{2}}{m^{2} \alpha^{2}}\right]\right. \\
& \left.-1-\sum_{j=1}^{D / 2} a_{j}(x) \frac{(1-D) \cdots(2 l-1-D)}{2^{j} m^{2 j}}\right\} .
\end{aligned}
$$

Further, we present the hyperbolic tangent function in this formula in the form $\tanh (x)=1-$ $2\left(e^{2 x}+1\right)^{-1}$. The product in curly braces of (9) multiplied by the first term in this representation is cancelled by the adiabatic subtraction terms [we have explicitly checked this for the terms involving $(m \alpha)^{-2}$ and $(m \alpha)^{-4}$; in the case $D=5$ these include all adiabatic terms] and the renormalized fermionic condensate in odd-dimensional dS spacetimes is presented in the form

$$
\langle\bar{\psi} \psi\rangle_{\mathrm{dS}, \text { ren }}=\frac{2 N m^{D}}{(4 \pi)^{(D+1) / 2}} \frac{\Gamma((1-D) / 2)}{e^{2 \pi m \alpha}+1} \prod_{j=0}^{D / 2-1}\left[1+\frac{(j+1 / 2)^{2}}{m^{2} \alpha^{2}}\right] .
$$

Note that the sign of the fermionic condensate is determined by the sign of the gamma function. For a massless fermionic field from (10) we find the following result:

$$
\langle\bar{\psi} \psi\rangle_{\mathrm{dS}, \text { ren }}=\frac{(-1)^{D / 2} N}{(4 \pi)^{(D+1) / 2} \alpha^{D}} \Gamma\left(\frac{D+1}{2}\right) .
$$


Having the fermionic condensate, we can evaluate the VEV of the energy-momentum tensor for the fermionic field in odd-dimensional dS spacetime by using the trace relation

$$
\left\langle T_{l}^{l}\right\rangle_{\mathrm{dS}, \mathrm{ren}}=m\langle\bar{\psi} \psi\rangle_{\mathrm{dS}, \mathrm{ren}} .
$$

Since the dS spacetime is maximally symmetric, we have $\left\langle T_{l}^{k}\right\rangle_{\mathrm{dS}, \text { ren }}=$ const $\delta_{l}^{k}$. The constant in this relation is found from (12). In this way, for even values $D$, for the renormalized VEV of the energy-momentum tensor we find:

$$
\left\langle T_{l}^{k}\right\rangle_{\mathrm{dS}, \text { ren }}=\frac{2 N m^{D+1} \delta_{l}^{k}}{(4 \pi)^{(D+1) / 2}(D+1)} \frac{\Gamma((1-D) / 2)}{e^{2 \pi m \alpha}+1} \prod_{j=0}^{D / 2-1}\left[1+\frac{(j+1 / 2)^{2}}{m^{2} \alpha^{2}}\right] .
$$

For a massless fermionic field this tensor vanishes. Of course, we could expect this result, since in odd-dimensional spacetimes the trace anomaly is absent. For large values of the mass, $m \alpha \gg 1$, the VEVs for both fermionic condensate and the energy-momentum tensor are exponentially suppressed. In particular, for 5-dimensional de Sitter spacetime formula (13) leads to the result

$$
\left\langle T_{l}^{k}\right\rangle_{\mathrm{dS}, \mathrm{ren}}=\frac{m^{5} \delta_{l}^{k}}{15 \pi^{2}} \frac{1}{e^{2 \pi m \alpha}+1}\left(1+\frac{5}{2 m^{2} \alpha^{2}}+\frac{9}{16 m^{4} \alpha^{4}}\right), D=4 .
$$

For $D=3$ the renormalized VEV of the energy-momentum tensor for fermionic field in dS spacetime is investigated in [18] (see also [10]). The corresponding expression has the form

$$
\left\langle T_{l}^{k}\right\rangle_{\mathrm{dS}, \mathrm{ren}}^{(D=3)}=\frac{\delta_{l}^{k}}{16 \pi^{2} \alpha^{4}}\left\{2 m^{2} \alpha^{2}\left(m^{2} \alpha^{2}+1\right)[\ln (m \alpha)-\operatorname{Re} \psi(i m \alpha)]+m^{2} \alpha^{2} / 6+11 / 60\right\},
$$

where $\psi(x)$ is the logarithmic derivative of the gamma-function. In the limit of zero mass, only the last term in curly braces contributes. For large values of the mass we have:

$$
\left\langle T_{l}^{k}\right\rangle_{\mathrm{dS}, \text { ren }}^{(D=3)} \approx-\frac{\delta_{l}^{k}}{960 \pi^{2} \alpha^{6} m^{2}}, m \alpha \gg 1,
$$

and, unlike to the case of odd dimensions, the suppression is power-law. In figure 1 we have

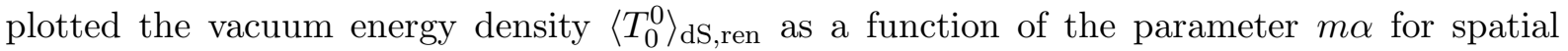
dimensions $D=3,4$.

In dS spacetime the energy-momentum tensor $\left\langle T_{l}^{k}\right\rangle_{\mathrm{dS} \text {,ren }}$ corresponds to a gravitational source of the cosmological constant type. Combining with the initial cosmological constant $\Lambda$, one-loop effects in uncompactified dS spacetime lead to the effective cosmological constant

$$
\Lambda_{\text {eff }}=\Lambda+8 \pi G_{D+1}\left\langle T_{0}^{0}\right\rangle_{\mathrm{dS}, \mathrm{ren}}
$$

where $G_{D+1}$ is the gravitational constant in $(D+1)$-dimensional spacetime. In the discussion above we have assumed that the quantum state of a fermionic field is the Bunch-Davies vacuum state (also called Euclidean vacuum). In [35] it was shown that for a scalar field with a wide range of mass and curvature coupling parameter the expectation values of the energy-momentum tensor in arbitrary physically admissable states approaches the expectation value in the BunchDavies vacuum at late times.

\section{Plane wave eigenspinors in toroidally compactified de Sitter spacetime}

Now we turn to the investigation of one-loop quantum effects induced by the compactness of the spatial dimensions in dS spacetime. We consider $(D+1)$-dimensional dS spacetime with 


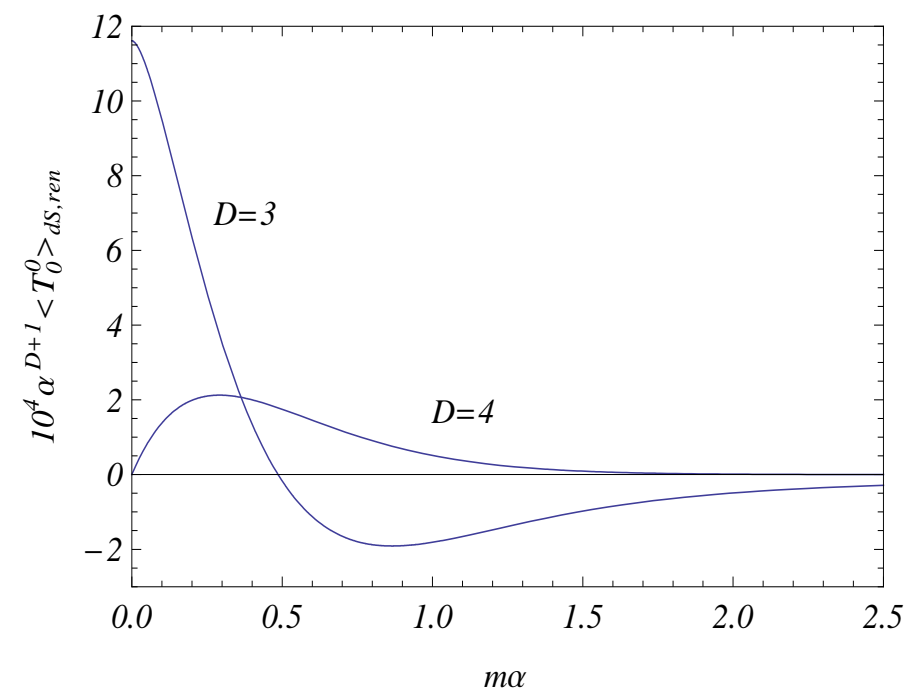

Figure 1: The vacuum energy density in uncompactified dS spacetime, $\left\langle T_{0}^{0}\right\rangle_{\mathrm{dS}, \text { ren }}$, as a function of the parameter $m \alpha$ for spatial dimensions $D=3,4$.

spatial topology $\mathrm{R}^{p} \times\left(\mathrm{S}^{1}\right)^{q}, p+q=D$. The compactification of the spatial dimensions leads to the modification of the spectrum for zero-point fluctuations of fields and as a result of this the VEVs of physical observables are changed. This is the topological Casimir effect which is well-investigated in the literature for various type of geometries.

In the $(D+1)$-dimensional flat spacetime the Dirac matrices are $N \times N$ matrices with $N=2^{[(D+1) / 2]}$ (see, for instance, [36]), where the square brackets mean the integer part of the enclosed expression. In the discussion below we will assume that these matrices are given in the chiral representation:

$$
\gamma^{(0)}=\left(\begin{array}{cc}
1 & 0 \\
0 & -1
\end{array}\right), \gamma^{(a)}=\left(\begin{array}{cc}
0 & \sigma_{a} \\
-\sigma_{a}^{+} & 0
\end{array}\right), a=1,2, \ldots, D
$$

From the anticommutation relations for the Dirac matrices one has $\sigma_{a} \sigma_{b}^{+}+\sigma_{b} \sigma_{a}^{+}=2 \delta_{a b}$. For example, in $D=4$ the first four matrices $\gamma^{(a)}, a=0,1,2,3$, can be taken the same as the corresponding matrices in 4-dimensional spacetime and $\gamma^{(4)}=\gamma^{(0)} \gamma^{(1)} \gamma^{(2)} \gamma^{(3)}$. In this case $\sigma_{1}, \sigma_{2}, \sigma_{3}$ are the standard Pauli matrices and

$$
\sigma_{4}=\left(\begin{array}{cc}
0 & -i \\
-i & 0
\end{array}\right)
$$

Note that, unlike to the Pauli matrices, $\sigma_{4}$ is antihermitian.

In order to evaluate the VEVs of the fermionic condensate and the energy-momentum tensor in toroidally compactified dS spacetime, we will use the direct mode-summation procedure. In this procedure we need to know a complete set of properly normalized eigenspinors $\left\{\psi_{\beta}^{(+)}, \psi_{\beta}^{(-)}\right\}$, specified by the collective index $\beta$. By virtue of spatial translation invariance the spatial part of the eigenfunctions $\psi_{\beta}^{( \pm)}$can be taken in the standard plane wave form $e^{ \pm i \mathbf{k z}}$, where $\mathbf{k}$ is the wave vector. We will decompose the vectors $\mathbf{z}$ and $\mathbf{k}$ into the components along the uncompactified and compactified dimensions, $\mathbf{z}=\left(\mathbf{z}_{p}, \mathbf{z}_{q}\right), \mathbf{k}=\left(\mathbf{k}_{p}, \mathbf{k}_{q}\right)$. One of the characteristic features of the field theory on backgrounds with non-trivial topology is the appearance of inequivalent types of fields with the same spin [37]. For fermion fields the boundary conditions along the 
compactified dimensions can be either periodic (untwisted field) or antiperiodic (twisted field). First we consider the field with periodicity conditions (no summation over $l$ ):

$$
\psi\left(t, \mathbf{z}_{p}, \mathbf{z}_{q}+L_{l} \mathbf{e}_{l}\right)=\psi\left(t, \mathbf{z}_{p}, \mathbf{z}_{q}\right),
$$

where $\mathbf{e}_{l}$ is the unit vector in the direction of the coordinate $z^{l}$ with the length $L_{l}, 0 \leqslant z^{l} \leqslant L_{l}$. The case of a fermionic field with antiperiodicity conditions will be discussed below. For a spinor field with periodicity conditions along the compactified dimensions the corresponding wave vector has the components

$$
\mathbf{k}_{q}=\left(2 \pi n_{p+1} / L_{p+1}, \ldots, 2 \pi n_{D} / L_{D}\right),
$$

where $n_{p+1}, \ldots, n_{D}=0, \pm 1, \pm 2, \ldots$

In order to find the time dependence of the fermionic eigenfunctions, we choose the basis tetrad in the form $e_{\mu}^{(0)}=\delta_{\mu}^{0}, e_{\mu}^{(a)}=e^{t / \alpha} \delta_{\mu}^{a}, a=1,2, \ldots, D$. For the components of the corresponding spin connection we have $\Gamma_{0}=0, \Gamma_{l}=\left(e^{t / \alpha} / 2 \alpha\right) \gamma^{(0)} \gamma^{(l)}, l=1,2, \ldots, D$. Decomposing the $N$-component spinor fields in the form

$$
\psi_{\beta}^{(+)}=e^{i \mathbf{k z}}\left(\begin{array}{c}
\varphi_{\beta,+}^{(+)} \\
\varphi_{\beta,-}^{(+)}
\end{array}\right),
$$

from the Dirac equation one finds the set of two first order differential equations:

$$
\begin{aligned}
\left(\eta \partial_{\eta}-D / 2-i \alpha m\right) \varphi_{\beta,+}^{(+)}-i \eta(\mathbf{k} \cdot \boldsymbol{\sigma}) \varphi_{\beta,-}^{(+)} & =0 \\
\left(\eta \partial_{\eta}-D / 2+i \alpha m\right) \varphi_{\beta,-}^{(+)}-i \eta\left(\mathbf{k} \cdot \boldsymbol{\sigma}^{+}\right) \varphi_{\beta,+}^{(+)} & =0
\end{aligned}
$$

where $\boldsymbol{\sigma}=\left(\sigma_{1}, \sigma_{2}, \ldots, \sigma_{D}\right)$. In these equations we have introduced a new independent variable $\eta$ in accordance with

$$
\eta=\alpha e^{-t / \alpha}, 0 \leqslant \eta<\infty .
$$

Note that $\tau=-\eta$ is the conformal time coordinate, in terms of which the dS line element (11) takes the conformally flat form.

By using the relation $(\mathbf{k} \cdot \boldsymbol{\sigma})\left(\mathbf{k} \cdot \boldsymbol{\sigma}^{+}\right)=k^{2}$, with

$$
k=\sqrt{\mathbf{k}_{p}^{2}+\mathbf{k}_{q}^{2}}
$$

from (23) we obtain the second order differential equations for the upper and lower components of the spinor:

$$
\left[\eta^{2} \partial_{\eta}^{2}-D \eta \partial_{\eta}+k^{2} \eta^{2}+\alpha^{2} m^{2} \pm i \alpha m+D(D+2) / 4\right] \varphi_{\beta, \pm}^{(+)}=0 .
$$

These equations are solved in terms of the Hankel functions and the corresponding solutions have the form

$$
\varphi_{\beta, \pm}^{(+)}=\eta^{(D+1) / 2}\left[C_{1, \pm} H_{ \pm 1 / 2-i \alpha m}^{(1)}(k \eta)+C_{2, \pm} H_{ \pm 1 / 2-i \alpha m}^{(2)}(k \eta)\right] .
$$

Different choices of the coefficients $C_{j, \pm}$ correspond to different vacuum states. As in section 2. we will assume that the fermionic field is prepared in the de Sitter invariant Bunch-Davies vacuum, for which $C_{2, \pm}=0$. Hence, for a fermionic field in the Bunch-Davies vacuum state the solutions to (27) are the functions

$$
\begin{aligned}
& \varphi_{\beta,+}^{(+)}=\varphi^{(c)} \eta^{(D+1) / 2} H_{1 / 2-i \alpha m}^{(1)}(k \eta), \\
& \varphi_{\beta,-}^{(+)}=-i \varphi^{(c)}(\mathbf{n} \cdot \boldsymbol{\sigma}) \eta^{(D+1) / 2} H_{-1 / 2-i \alpha m}^{(1)}(k \eta),
\end{aligned}
$$


where $\varphi^{(c)}$ is an arbitrary constant spinor and $\mathbf{n}=\mathbf{k} / k$. Note that, in order to obtain the relation between the coefficients in (29) we have used equation (23).

Now, we can construct the positive frequency solutions to the Dirac equation on the base of functions (29):

$$
\psi_{\beta}^{(+)}=A_{\beta} \eta^{(D+1) / 2} e^{i \mathbf{k} \cdot \mathbf{r}}\left(\begin{array}{c}
H_{1 / 2-i \alpha m}^{(1)}(k \eta) w_{\sigma}^{(+)} \\
-i(\mathbf{n} \cdot \boldsymbol{\sigma}) H_{-1 / 2-i \alpha m}^{(1)}(k \eta) w_{\sigma}^{(+)}
\end{array}\right)
$$

where $\beta=(\mathbf{k}, \sigma)$, and $w_{\sigma}^{(+)}, \sigma=1, \ldots, N / 2$, are one-column matrices having $N / 2$ rows with the elements $w_{l}^{(\sigma)}=\delta_{l \sigma}$. In the similar way, for the negative frequency solutions we find

$$
\psi_{\beta}^{(-)}=A_{\beta} \eta^{(D+1) / 2} e^{-i \mathbf{k} \cdot \mathbf{r}}\left(\begin{array}{c}
i(\mathbf{n} \cdot \boldsymbol{\sigma}) H_{-1 / 2+i \alpha m}^{(2)}(k \eta) w_{\sigma}^{(-)} \\
H_{1 / 2+i \alpha m}^{(2)}(k \eta) w_{\sigma}^{(-)}
\end{array}\right),
$$

with $w_{\sigma}^{(-)}=i w_{\sigma}^{(+)}$.

From the orthonormalization condition for the eigenfunctions (30) and (31), for the coefficient $A_{\beta}$ one obtains

$$
A_{\beta}^{2}=\frac{k e^{\pi \alpha m}}{2^{p+2} \pi^{p-1} V_{q} \alpha^{D}},
$$

where $V_{q}=L_{p+1} \cdots L_{D}$ is the volume of the compactified subspace. For a massless fermionic field the Hankel functions are expressed in terms of exponentials and we have the standard conformal relation $\psi_{\beta}^{( \pm)}=(\eta / \alpha)^{(D+1) / 2} \psi_{\beta}^{(\mathrm{M})( \pm)}$ between eigenspinors (30), (31) defining the Bunch-Davies vacuum in dS spacetime and the corresponding eigenspinors $\psi_{\beta}^{(\mathrm{M})( \pm)}$ for the Minkowski spacetime with spatial topology $\mathrm{R}^{p} \times\left(\mathrm{S}^{1}\right)^{q}$.

The plane wave eigenspinors for a twisted spinor field are constructed in a similar way. For this field we have the antiperiodicity conditions along the compactified dimensions:

$$
\psi\left(t, \mathbf{z}_{p}, \mathbf{z}_{q}+L_{l} \mathbf{e}_{l}\right)=-\psi\left(t, \mathbf{z}_{p}, \mathbf{z}_{q}\right) .
$$

The corresponding eigenfunctions are given by formulae (30), (311), where now the components of the wave vector along the compactified dimensions are given by the formula

$$
\mathbf{k}_{q}=\left(\pi\left(2 n_{p+1}+1\right) / L_{p+1}, \ldots, \pi\left(2 n_{D}+1\right) / L_{D}\right),
$$

with $n_{p+1}, \ldots, n_{D}=0, \pm 1, \pm 2, \ldots$, and

$$
k^{2}=k_{p}^{2}+\sum_{l=p+1}^{D}\left[\pi\left(2 n_{l}+1\right) / L_{l}\right]^{2}
$$

Note that the physical wave vector is given by the combination $\mathrm{k} \eta / \alpha$.

\section{Fermionic condensate in toroidally compactified de Sitter spacetime}

In this and in the following sections we investigate the fermionic vacuum effects induced by the non-trivial topology of the dS spacetime. The scheme of the corresponding calculations is similar to that for the case of $\mathrm{dS}_{4}$ given in [33], and we will omit the details. Expanding the field 
operator in terms of the complete set of eigenfunctions, described in the previous section, and using the commutation relations, we find the mode-sum formula for the fermionic condensate:

$$
\langle\bar{\psi} \psi\rangle_{p, q}=\int d \mathbf{k}_{p} \sum_{\mathbf{k}_{q}, \sigma} \bar{\psi}_{\mathbf{k}, \sigma}^{(-)} \psi_{\mathbf{k}, \sigma}^{(-)},
$$

where $\bar{\psi}_{\mathbf{k}, \sigma}^{(-)}=\psi_{\mathbf{k}, \sigma}^{(-)+} \gamma^{0}$ is the Dirac adjoint. Applying to the sum over $n_{p+1}$ in (36) the AbelPlana formula (see, for instance, [38]), we find the following recurrence formula for the fermionic condensate in dS spacetime with spatial topology $\mathrm{R}^{p} \times\left(\mathrm{S}^{1}\right)^{q}$ :

$$
\langle\bar{\psi} \psi\rangle_{p, q}=\langle\bar{\psi} \psi\rangle_{p+1, q-1}+\Delta_{p+1}\langle\bar{\psi} \psi\rangle_{p, q}
$$

where $\langle\bar{\psi} \psi\rangle_{p+1, q-1}$ is the fermionic condensate in dS spacetime having topology $\mathrm{R}^{p+1} \times\left(\mathrm{S}^{1}\right)^{q-1}$, and the term

$$
\begin{aligned}
\Delta_{p+1}\langle\bar{\psi} \psi\rangle_{p, q}= & \frac{8 N \eta^{D+1} \alpha^{-D} L_{p+1}}{(2 \pi)^{(p+3) / 2} V_{q}} \sum_{n=1}^{\infty} \sum_{n_{p+2}=-\infty}^{+\infty} \cdots \sum_{n_{D}=-\infty}^{+\infty} \int_{0}^{\infty} d x x^{2} \\
& \times \frac{\operatorname{Im}\left[K_{1 / 2-i \alpha m}(\eta x) I_{1 / 2+i \alpha m}(\eta x)\right]}{\left(n L_{p+1}\right)^{p-1}} f_{(p-1) / 2}\left(n L_{p+1} \sqrt{x^{2}+k_{\mathbf{n}_{q-1}}^{2}}\right),
\end{aligned}
$$

is induced by the compactness of the $(p+1)$ th dimension. In Eq. (38), $I_{\nu}(x)$ and $K_{\nu}(x)$ are the modified Bessel functions and we have introduced the notations

$$
f_{\nu}(x) \equiv x^{\nu} K_{\nu}(x), k_{\mathbf{n}_{q-1}}^{2}=\sum_{l=p+2}^{D}\left(2 \pi n_{l} / L_{l}\right)^{2} .
$$

Note that for a massless field the topological part in the fermionic condensate vanishes.

On the base of the recurrence relation (37) the fermionic condensate can be decomposed as

$$
\langle\bar{\psi} \psi\rangle_{p, q}=\langle\bar{\psi} \psi\rangle_{\mathrm{dS}, \mathrm{ren}}+\langle\bar{\psi} \psi\rangle_{\mathrm{c}},\langle\bar{\psi} \psi\rangle_{\mathrm{c}}=\sum_{l=1}^{q} \Delta_{D+1-l}\langle\bar{\psi} \psi\rangle_{D-l, l}
$$

where the fermionic condensate in uncompactified $\mathrm{dS}$ spacetime, $\langle\bar{\psi} \psi\rangle_{\mathrm{dS} \text {,ren }}$, is given by formula (10) and the part $\langle\bar{\psi} \psi\rangle_{\mathrm{c}}$ is induced by the non-trivial spatial topology. Note that the expression on the right of formula (38) is finite and the renormalization is needed for the uncompactified dS part only. Of course, we could expect the finiteness of the topological part, since the toroidal compactification does not change the local geometry and, hence, the structure of the divergences is the same as in uncompactified dS spacetime. From formula (38) we see that the topological part depends on the variable $\eta$ and the length scales $L_{l}$ in the combinations $L_{l} / \eta$. Noting that $a(\eta) L_{l}$ is the comoving length with $a(\eta)=\alpha / \eta$ being the scale factor, we conclude that the topological part of the fermionic condensate is a function of the comoving lengths of the compactified dimensions.

The general formula for the topological part in the fermionic condensate is simplified in the asymptotic regions of the parameters. In the limit when the comoving length of the $(p+1)$ th dimension is much smaller than the dS curvature radius, $a(\eta) L_{p+1} \ll \alpha$, the leading term in the asymptotic expansion of the fermionic condensate has the form

$$
\Delta_{p+1}\langle\bar{\psi} \psi\rangle_{p, q} \approx-\frac{2 N(\eta / \alpha)^{D-1} m}{(2 \pi)^{p / 2+1} L_{p+1}^{p-1} V_{q}} \sum_{n=1}^{\infty} \sum_{n_{p+2}=-\infty}^{+\infty} \cdots \sum_{n_{D}=-\infty}^{+\infty} \frac{f_{p / 2}\left(n L_{p+1} k_{\mathbf{n}_{q-1}}\right)}{n^{p}} .
$$


In this limit the topological part is negative. Taking into account the relation between the conformal and synchronous time coordinates, we see that formula (41) describes the asymptotic behavior in the early stages of the cosmological expansion corresponding to $t \rightarrow-\infty$. Since the part $\langle\bar{\psi} \psi\rangle_{\mathrm{dS} \text {,ren }}$ in the fermionic condensate is time-independent, we conclude that in this limit the topological part dominates.

Now let us consider large values of the comoving compactification scale, $a(\eta) L_{p+1} \gg \alpha$. In terms of the synchronous time coordinate this corresponds to the late stages of the cosmological evolution, $t \rightarrow+\infty$. By using the formulae for the modified Bessel functions for small values of the arguments, we find the leading term given below:

$$
\Delta_{p+1}\langle\bar{\psi} \psi\rangle_{p, q} \approx-\frac{N \alpha B_{0} \sin \left[2 m t-2 \alpha m \ln \left(\alpha / L_{p+1}\right)-\phi_{0}\right]}{2^{p / 2-1} \pi^{(p+1) / 2} L_{p+1}^{p+1} V_{q} \cosh (\alpha m \pi) e^{(D+1) t / \alpha}} .
$$

The constants in the expression on the right-hand side are defined by the relation

$$
B_{0} e^{i \phi_{0}}=\frac{2^{-i \alpha m}}{\Gamma(1 / 2+i \alpha m)} \sum_{n=1}^{\infty} \sum_{n_{p+2}=-\infty}^{+\infty} \cdots \sum_{n_{D}=-\infty}^{+\infty} \frac{f_{p / 2+1+i \alpha m}\left(n L_{p+1} k_{\mathbf{n}_{q-1}}\right)}{n^{p+2+2 i \alpha m}} .
$$

Hence, in the limit when the comoving length of the compactified dimensions is much larger than the curvature radius of dS spacetime, for a massive fermionic field the topological part oscillates with the amplitude exponentially decreasing with respect to the synchronous time coordinate. The damping factor in the amplitude and the oscillation frequency are the same for all terms in the sum over $l$ in the expression (40) for $\langle\bar{\psi} \psi\rangle_{\mathrm{c}}$ and we have the similar oscillating behavior for the total topological term: $\langle\bar{\psi} \psi\rangle_{c} \propto e^{-(D+1) t / \alpha} \sin \left(2 m t+\phi_{\mathrm{c}}\right)$.

In the case of a fermionic field with antiperiodicity conditions (33), the fermionic condensate can be found in a way similar to that for the periodicity conditions. It can be seen that the corresponding formulae for the topological parts are obtained from those for the field with periodicity conditions inserting the factor $(-1)^{n}$ in the summation over $n$ and replacing the definition for $k_{\mathbf{n}_{q-1}}^{2}$ by

$$
k_{\mathbf{n}_{q-1}}^{2}=\sum_{l=p+2}^{D}\left[\pi\left(2 n_{l}+1\right) / L_{l}\right]^{2} .
$$

In situations where the main contribution comes from the term $n=1$, the topological parts in the fermionic condensate for fields with periodicity and antiperiodicity conditions have opposite signs.

\section{Vacuum expectation value of the energy-momentum tensor}

The one-loop topological effects in the VEV of the energy-momentum tensor are investigated in a way similar to that for the fermionic condensate. The corresponding mode-sum has the form

$$
\left\langle T_{\mu \nu}\right\rangle=\frac{i}{2} \int d \mathbf{k}_{p} \sum_{\mathbf{k}_{q}, \sigma}\left[\bar{\psi}_{\mathbf{k}, \sigma}^{(-)} \gamma_{(\mu} \nabla_{\nu)} \psi_{\mathbf{k}, \sigma}^{(-)}-\left(\nabla_{(\mu} \bar{\psi}_{\mathbf{k}, \sigma}^{(-)}\right) \gamma_{\nu)} \psi_{\mathbf{k}, \sigma}^{(-)}\right]
$$

For a fermionic field with periodic boundary conditions, substituting the eigenfunctions (31) into this formula and by using the Abel-Plana formula, the VEV of the energy-momentum tensor in dS spacetime with spatial topology $\mathrm{R}^{p} \times\left(\mathrm{S}^{1}\right)^{q}$ is presented in the decomposed form

$$
\left\langle T_{k}^{l}\right\rangle_{p, q}=\left\langle T_{k}^{l}\right\rangle_{p+1, q-1}+\Delta_{p+1}\left\langle T_{k}^{l}\right\rangle_{p, q}
$$


Here $\left\langle T_{k}^{l}\right\rangle_{p+1, q-1}$ is the VEV of the energy-momentum tensor for the topology $\mathrm{R}^{p+1} \times\left(\mathrm{S}^{1}\right)^{q-1}$ and the part (no summation over $l$ )

$$
\begin{aligned}
\Delta_{p+1}\left\langle T_{k}^{l}\right\rangle_{p, q}= & \frac{N \eta^{D+2} \alpha^{-D-1} L_{p+1} \delta_{k}^{l}}{(2 \pi)^{(p+1) / 2} V_{q}} \sum_{n=1}^{\infty} \sum_{n_{p+2}=-\infty}^{+\infty} \ldots \sum_{n_{D}=-\infty}^{+\infty} \int_{0}^{\infty} d x x \\
& \times \frac{\operatorname{Re}\left[I_{-1 / 2-i \alpha m}^{2}(\eta x)-I_{1 / 2+i \alpha m}^{2}(\eta x)\right]}{\cosh (\alpha m \pi)\left(L_{p+1} n\right)^{p+1}} f_{p}^{(l)}\left(n L_{p+1} \sqrt{x^{2}+k_{\mathbf{n}_{q-1}}^{2}}\right),
\end{aligned}
$$

is due to the compactness of the $(p+1)$ th dimension. For the separate components the functions $f_{p}^{(l)}(y)$ have the form

$$
\begin{aligned}
f_{p}^{(l)}(y) & =f_{(p+1) / 2}(y), l=0,1, \ldots, p \\
f_{p}^{(p+1)}(y) & =-\left[p f_{(p+1) / 2}(y)+y^{2} f_{(p-1) / 2}(y)\right], \\
f_{p}^{(l)}(y) & =k_{l}^{2}\left(n L_{p+1}\right)^{2} f_{(p-1) / 2}(y), l=p+2, \ldots, D,
\end{aligned}
$$

where $k_{l}=2 \pi n_{l} / L_{l}$ and the function $f_{\nu}(x)$ is defined in (39). The topological parts (47) are finite and by the recurrence relation (46) the renormalization procedure is reduced to the renormalization of the corresponding VEV in uncompactified dS spacetime. It can be seen that the topological part satisfies the trace relation $\Delta_{p+1}\left\langle T_{l}^{l}\right\rangle_{p, q}=m \Delta_{p+1}\langle\bar{\psi} \psi\rangle_{p, q}$ and is covariantly conserved in dS spacetime: $\left(\Delta_{p+1}\left\langle T_{l}^{k}\right\rangle_{p, q}\right)_{; k}=0$. In particular, this part is traceless for a massless field. In the uncompactified subspace the equation of state for the topological part of the energy-momentum tensor is of the cosmological constant type. Note that the topological parts are time-dependent and they break the dS symmetry.

After the repetitive application of the recurrence formula (46), the VEV of the energymomentum tensor for the topology $\mathrm{R}^{p} \times\left(\mathrm{S}^{1}\right)^{q}$ is presented in the form

$$
\left\langle T_{l}^{k}\right\rangle_{p, q}=\left\langle T_{l}^{k}\right\rangle_{\mathrm{dS}, \mathrm{ren}}+\left\langle T_{l}^{k}\right\rangle_{\mathrm{c}},\left\langle T_{l}^{k}\right\rangle_{\mathrm{c}}=\sum_{l=1}^{q} \Delta_{D+1-l}\left\langle T_{l}^{k}\right\rangle_{D-l, l}
$$

where the renormalized VEV in the uncompactified dS spacetime, $\left\langle T_{l}^{k}\right\rangle_{\mathrm{dS}, \text { ren }}$, is given by formula (13) and $\left\langle T_{l}^{k}\right\rangle_{\mathrm{c}}$ is the topological part.

For a massless fermionic field, from (47) one finds (no summation over $l$ )

$$
\Delta_{p+1}\left\langle T_{l}^{l}\right\rangle_{p, q}=\frac{2 N(\eta / \alpha)^{D+1}}{(2 \pi)^{p / 2+1} V_{q} L_{p+1}^{p+1}} \sum_{n=1}^{\infty} \sum_{n_{p+2}=-\infty}^{+\infty} \cdots \sum_{n_{D}=-\infty}^{+\infty} \frac{g_{p}^{(l)}\left(n L_{p+1} k_{\mathbf{n}_{q-1}}\right)}{n^{p+2}}
$$

with the notations

$$
\begin{aligned}
g_{p}^{(l)}(y) & =f_{p / 2+1}(y), l=0,1, \ldots, p, \\
g_{p}^{(p+1)}(y) & =-(p+1) f_{p / 2+1}(y)-y^{2} f_{p / 2}(y), \\
g_{p}^{(l)}(y) & =\left(n L_{p+1} k_{l}\right)^{2} f_{p / 2}(y), l=p+2, \ldots, D .
\end{aligned}
$$

The massless fermionic field is conformally invariant in any dimension and in this case the problem under consideration is conformally related to the corresponding problem in the Minkowski spacetime with spatial topology $\mathrm{R}^{p} \times\left(\mathrm{S}^{1}\right)^{q}$. Formula (50) is also obtained from the relation $\Delta_{p+1}\left\langle T_{i}^{i}\right\rangle_{p, q}=a^{-D-1}(\eta) \Delta_{p+1}\left\langle T_{i}^{i}\right\rangle_{p, q}^{(\mathrm{M})}$, with $a(\eta)$ being the scale factor. Comparing expression (50) with the corresponding formula from [30] for a conformally coupled massless scalar field, we see that the following relation takes place: $\Delta_{p+1}\left\langle T_{k}^{l}\right\rangle_{p, q}=-N \Delta_{p+1}\left\langle T_{k}^{l}\right\rangle_{p, q}^{\text {(scalar) }}$. 
Now we consider the behavior of the vacuum energy-momentum tensor in the asymptotic regions of the parameters. For small values of the comoving length $a(\eta) L_{p+1}$ with respect to the dS curvature radius, $a(\eta) L_{p+1} \ll \alpha$, to the leading order the topological part in the VEV of the energy-momentum tensor coincides with that for a massless field given by formula (50). In particular, the topological part of the vacuum energy density is positive. This limit corresponds to the early stages of the cosmological evolution, $t \rightarrow-\infty$, and at these stages the total VEV is dominated by the topological part. In this limit the back-reaction effects of the topological terms are important and these effects can change the dynamics essentially (for a discussion of back-reaction effects from vacuum fluctuations on the dynamics of dS spacetime see, for instance, [39] and references therein).

For large values of the comoving length, $a(\eta) L_{p+1} \gg \alpha$, the leading term in the topological part is given by the formula (no summation over $l$ ):

$$
\Delta_{p+1}\left\langle T_{l}^{l}\right\rangle_{p, q} \approx \frac{N B_{l} \cos \left[2 m t-2 \alpha m \ln \left(\alpha / L_{p+1}\right)-\phi_{l}\right]}{2^{p / 2} \pi^{(p+1) / 2} V_{q} L_{p+1}^{p+1} \cosh (\alpha m \pi) e^{(D+1) t / \alpha}} .
$$

Here $B_{l}, \phi_{l}$ for $l=0,1, \ldots, p$, are given by relation (43), and $B_{l}, \phi_{l}, l=p+1, \ldots, D$, are defined by the formula

$$
\begin{aligned}
B_{l} e^{i \phi_{l}}= & \frac{-2^{-i \alpha m}}{\Gamma(1 / 2+i \alpha m)} \sum_{n=1}^{\infty} \sum_{n_{p+2}=-\infty}^{+\infty} \cdots \sum_{n_{D}=-\infty}^{+\infty} \frac{1}{n^{p+2+2 i \alpha m}} \\
& \times\left[(p+1+2 i \alpha m) f_{p / 2+1+i \alpha m}(x)+x^{2} f_{p / 2+i \alpha m}(x)\right]_{x=n L_{p+1} k_{\mathbf{n}_{q-1}}},
\end{aligned}
$$

for $l=p+1$, and by the formula

$$
B_{l} e^{i \phi_{l}}=\frac{2^{-i \alpha m} L_{p+1}^{2}}{\Gamma(1 / 2+i \alpha m)} \sum_{n=1}^{\infty} \sum_{n_{p+2}=-\infty}^{+\infty} \cdots \sum_{n_{D}=-\infty}^{+\infty} k_{l}{ }^{2} \frac{f_{p / 2+i \alpha m}\left(n L_{p+1} k_{\mathbf{n}_{q-1}}\right)}{n^{p+2 i \alpha m}},
$$

for $l=p+2, \ldots, D$. Formula (52) describes the asymptotic behavior of the topological part in the late stages of cosmological evolution corresponding to the limit $t \rightarrow+\infty$. In this limit the behavior of the topological part for a massive spinor field is damping oscillatory. As in the case of the fermionic condensate, the damping factor in the amplitude and the oscillation frequency are the same for all terms in the sum of Eq. (49) and the total topological term behaves like $\left\langle T_{l}^{k}\right\rangle_{\mathrm{c}} \propto e^{-(D+1) t / \alpha} \cos \left(2 m t+\phi_{\mathrm{c}}^{\prime}\right)$. As the vacuum energy-momentum tensor for uncompactified dS spacetime is time-independent, we have similar damping oscillations in the total energy-momentum tensor $\left\langle T_{l}^{k}\right\rangle_{\mathrm{dS} \text {,ren }}+\left\langle T_{l}^{k}\right\rangle_{\mathrm{c}}$. This type of oscillations are absent in the case of a massless field when the topological parts decay monotonically as $e^{-(D+1) t / \alpha}$.

The topological parts in the VEV of the energy-momentum tensor for the fermionic field with antiperiodicity conditions along the compactified dimensions are investigated in the way similar to that for untwisted field. The corresponding formulae for the topological parts are obtained from the expressions for the field with periodicity conditions inserting the factor $(-1)^{n}$ in the summation over $n$ and replacing the definition for $k_{\mathbf{n}_{q-1}}^{2}$ by (44).

In figure 2 we have plotted the topological parts in the VEVs of the energy density (left panel) and the vacuum stress along the compactified dimension (right panel) as functions of the ratio $L / \eta$ for the value of the parameter $\alpha m=4$ and in the special case of $\mathrm{dS}_{5}$ having spatial topology $\mathrm{R}^{3} \times \mathrm{S}^{1}$ with the length of the compactified dimension $L_{4}=L$. This topology corresponds to the original Kaluza-Klein model [40]. Note that the ratio $L / \eta=a(\eta) L / \alpha$ is the comoving length of the compactified dimension in units of the dS curvature radius. Full/dashed curves correspond 
to the fields with periodicity/antiperiodicity conditions along the compactified dimension. Note that for the massless fermionic field in dS spacetime with the spatial topology $\mathrm{R}^{3} \times \mathrm{S}^{1}$ one has

$$
\left\langle T_{k}^{l}\right\rangle_{\mathrm{c}, m=0}=\frac{b \zeta(5)}{\pi^{2}}\left(\frac{\eta}{\alpha L}\right)^{5} \operatorname{diag}(1,1,1,1,-4),
$$

where $b=3$ for the field with periodicity condition and $b=-45 / 16$ for the field with antiperiodicity condition, $\zeta(x)$ is the Riemann zeta function. As we have explained before, in the limit $L / \eta \ll 1$, the tensor (55) is the leading term in the corresponding asymptotic expansion for the VEV of the energy-momentum tensor of the massive field.
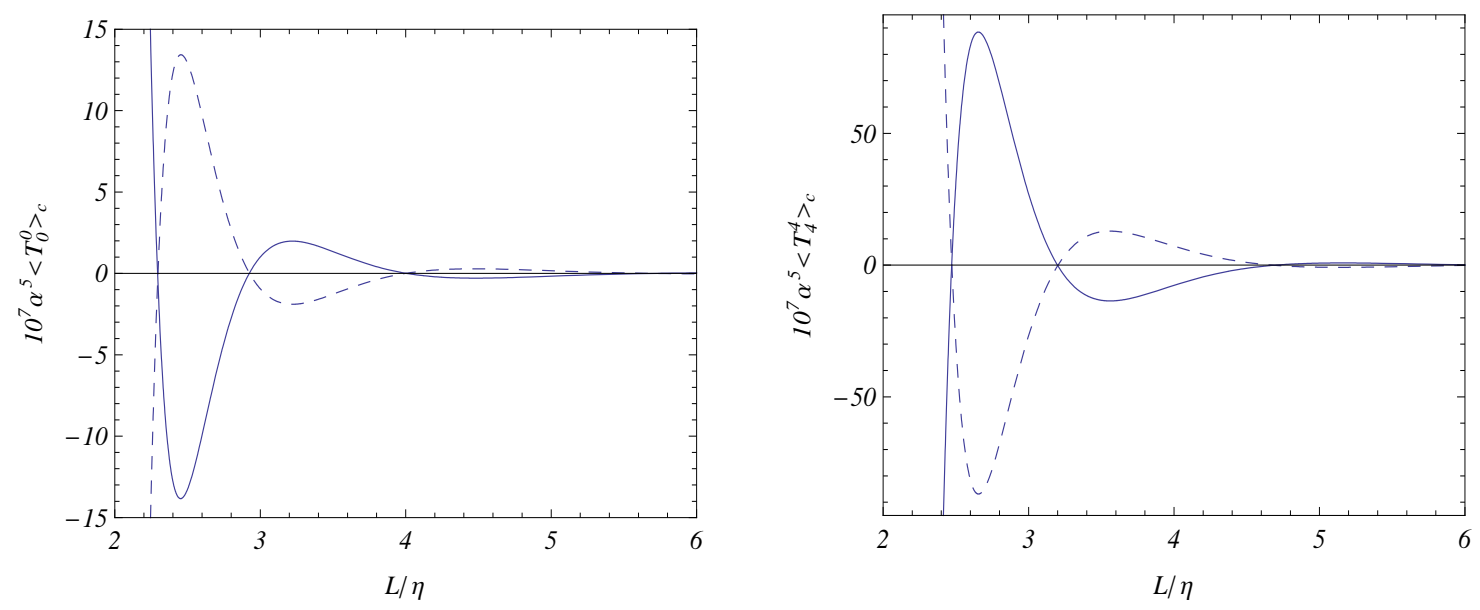

Figure 2: The topological parts in the VEVs of the energy density (left panel) and the vacuum stress along the compactified dimension (right panel) as functions of the ratio $L / \eta$ for the value of the parameter $\alpha m=4$ and in the special case of spatial topology $\mathrm{R}^{3} \times \mathrm{S}^{1}$. Full/dashed curves correspond to fields with periodicity/antiperiodicity conditions along the compactified dimension.

In order to illustrate the dependence of the topological parts on the mass, in figure 3 the ratio $\left\langle T_{0}^{0}\right\rangle_{\mathrm{c}} /\left\langle T_{0}^{0}\right\rangle_{\mathrm{c}, m=0}$ is plotted as a function of the parameter $\alpha m$ for fermionic field with periodicity conditions in $\mathrm{dS}_{5}$ with topology $\mathrm{R}^{3} \times \mathrm{S}^{1}$. The numbers near the curves correspond to the values of the ratio $L / \eta$ with $L$ being the length of the compactified dimension. In accordance with formula (52), for large values of the mass the topological part is an oscillating function. The amplitude of these oscillations is exponentially suppressed and in the given scale they are seen only for the case $L / \eta=4$.

\section{Conclusion}

In the present paper we have investigated the fermionic condensate and the VEV of the energymomentum tensor for a massive fermionic field in higher-dimensional dS spacetime with toroidally compactified spatial dimensions. In Section 2 we have considered the corresponding quantities in uncompactified odd-dimensional dS spacetime assuming that the field is prepared in the BunchDavies vacuum state. The renormalization is done by using the dimensional regularization procedure. Closed expressions, formulae (10), (13), are derived for the renormalized fermionic condensate and the VEV of the energy-momentum tensor. For large values of the mass these quantities are exponentially suppressed. Note that in even-dimensional dS spacetime for large massless the suppression is power-law. 


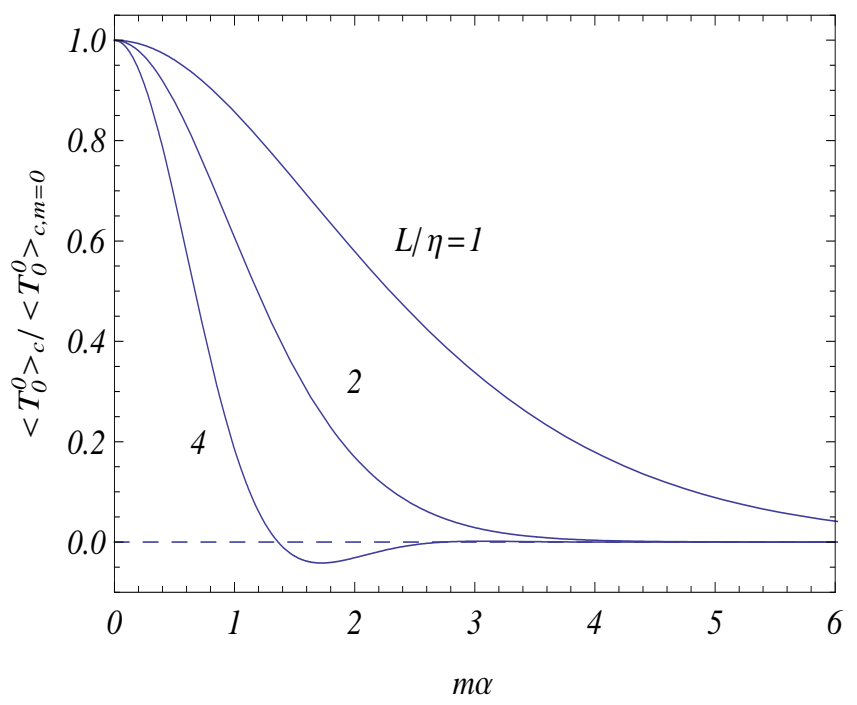

Figure 3: The ratio $\left\langle T_{0}^{0}\right\rangle_{\mathrm{c}} /\left\langle T_{0}^{0}\right\rangle_{\mathrm{c}, m=0}$ as a function of the parameter $\alpha m$ for a fermionic field with periodicity conditions in $\mathrm{dS}_{5}$ with spatial topology $\mathrm{R}^{3} \times \mathrm{S}^{1}$. The numbers near the curves correspond to the values of the ratio $L / \eta$.

Further, we have investigated one-loop quantum effects on the fermionic vacuum induced by the non-trivial topology of spatial dimensions. Specifically, we have considered the dS spacetime with toroidally compactified dimensions having the spatial topology $\mathrm{R}^{p} \times\left(\mathrm{S}^{1}\right)^{q}$. For the evaluation of the vacuum densities the mode-summation procedure is employed. In this procedure we need to know the corresponding eigenspinors satisfying appropriate boundary conditions along the compactified dimensions. These eigenspinors are constructed in section 3 for both fields with periodicity and antiperiodicity boundary conditions. By using these eigenfunctions and applying to the mode-sums Abel-Plana formula, the VEVs for the spatial topology $\mathrm{R}^{p} \times\left(\mathrm{S}^{1}\right)^{q}$ are presented in the form of the sum of the corresponding quantity in the topology $\mathrm{R}^{p+1} \times\left(\mathrm{S}^{1}\right)^{q-1}$ and of the part which is induced by the compactness of $(p+1)$ th dimension. For the field with periodicity conditions the topological parts are given by formulae (38) and (47). The corresponding formulae for the field with antiperiodicity conditions are obtained from those for the field with periodicity conditions inserting the factor $(-1)^{n}$ in the summation over $n$ and replacing the definition for $k_{\mathbf{n}_{q-1}}^{2}$ by (44).

The topological parts are finite and the renormalization procedure is reduced to that for the uncompactified dS spacetime. These parts are time-dependent and break the dS symmetry. The corresponding vacuum stresses along the uncompactified dimensions coincide with the energy density and, hence, in the uncompactified subspace the equation of state for the topological part of the energy-momentum tensor is of the cosmological constant type. For a massless fermionic field the problem under consideration is conformally related to the corresponding problem in the Minkowski spacetime with spatial topology $\mathrm{R}^{p} \times\left(\mathrm{S}^{1}\right)^{q}$ and the topological part of the fermionic condensate vanishes. For the VEV of the energy-momentum tensor we have the standard relation $\left\langle T_{k}^{l}\right\rangle_{c}=a^{-(D+1)}(\eta)\left\langle T_{k}^{l}\right\rangle_{c}^{(\mathrm{M})}$ between the topological contributions.

For a massive fermionic field, in the limit when the comoving length of a compactified dimension is much smaller than the dS curvature radius, the topological part in the VEV of the energy-momentum tensor coincides with the corresponding quantity for a massless field and is conformally related to the VEV in toroidally compactified Minkowski spacetime. In particular, the topological part in the vacuum energy density is positive for an untwisted fermionic field. 
This limit corresponds to the early stages of the cosmological evolution and the topological parts dominate over the uncompactified dS parts. At these stages the back-reaction effects of the topological terms are important and these effects can essentially change the dynamics of the model. In the opposite limit, when the comoving lengths of the compactified dimensions are large with respect to the $\mathrm{dS}$ curvature radius, in the case of a massive field the asymptotic behavior of the topological parts is damping oscillatory for both fermionic condensate and the energymomentum tensor and to the leading order is given by formulae (42), (52). These formulae describe the behavior of the topological parts in the late stages of the cosmological expansion.

As the uncompactified dS parts are time-independent, we have similar oscillations in the total VEVs as well. Note that this type of oscillatory behavior is absent for a massless fermionic field.

\section{Acknowledgments}

E.R.B.M. thanks Conselho Nacional de Desenvolvimento Científico e Tecnológico (CNPq) for partial financial support, FAPESQ-PB/CNPq (PRONEX) and FAPES-ES/CNPq (PRONEX). A.A.S. was supported by the Armenian Ministry of Education and Science Grant No. 119 and by Conselho Nacional de Desenvolvimento Científico e Tecnológico (CNPq).

\section{References}

[1] A.D. Linde, Particle Physics and Inflationary Cosmology (Harwood Academic Publishers, Chur, Switzerland 1990).

[2] A.G. Riess et al., Astrophys. J. 659, 98 (2007); D.N. Spergel et al., Astrophys. J. Suppl. Ser. 170, 377 (2007); U. Seljak, A. Slosar, and P. McDonald, JCAP 0610, 014 (2006); E. Komatsu et al., arXiv:0803.0547.

[3] A. Linde, JCAP 10, 004 (2004).

[4] B. McInnes, Nucl. Phys. B 709, 213 (2005); B. McInnes, Nucl. Phys. B 748 , 309 (2006).

[5] Y.B. Zeldovich and A.A. Starobinsky, Sov. Astron. Lett. 10, 135 (1984).

[6] Yu.P. Goncharov and A.A. Bytsenko, Phys. Lett. B 160, 385 (1985); Yu.P. Goncharov and A.A. Bytsenko, Phys. Lett. B 169, 171 (1986); Yu.P. Goncharov and A.A. Bytsenko, Nucl. Phys. B 271, 726 (1986); Yu.P. Goncharov and A.A. Bytsenko, Class. Quant. Grav. 4, 555 (1987).

[7] L.H. Ford, Phys. Rev. D 22, 3003 (1980).

[8] L.H. Ford and T. Yoshimura, Phys. Lett. A 70, 89 (1979); D.J. Toms, Phys. Rev. D 21, 928 (1980).

[9] D.J. Toms, Phys. Rev. D 21, 2805 (1980); S.D. Odintsov, Sov. J. Nucl. Phys. 48, 1148 (1988); I.L. Buchbinder and S.D. Odintsov, Int. J. Mod. Phys. A 4, 4337 (1989); Fortschr. Phys. 37, 225 (1989); I.L. Buchbinder, S.D. Odintsov, and V.P. Dergaleo, Theor. Math. Phys. 80, 150 (1989).

[10] A.A. Grib, S.G. Mamayev, and V.M. Mostepanenko, Vacuum Quantum Effects in Strong Fields (St. Petersburg, Friedmann Laboratory Publishing, 1994). 
[11] V.M. Mostepanenko and N.N. Trunov, The Casimir Effect and Its Applications (Clarendon, Oxford, 1997); M. Bordag, U. Mohidden, and V.M. Mostepanenko, Phys. Rep. 353, 1 (2001); K.A. Milton, The Casimir Effect: Physical Manifestation of Zero-Point Energy (World Scientific, Singapore, 2002); E. Elizalde, S.D. Odintsov, A. Romeo, A.A. Bytsenko and S. Zerbini, Zeta regularization techniques with applications (World Scientific, Singapore, 1994); M.J. Duff, B.E.W. Nilsson, and C.N. Pope, Phys. Rep. 130, 1 (1986); A.A. Bytsenko, G. Cognola, L. Vanzo, and S. Zerbini, Phys. Rep. 266, 1 (1996).

[12] K.A. Milton, Grav. Cosmol. 9, 66 (2003); E. Elizalde, J. Phys. A 39, 6299 (2006); B. Green and J. Levin, JHEP 11, 096 (2007); P. Burikham, A. Chatrabhuti, P. Patcharamaneepakorn, and K. Pimsamarn, JHEP 07, 013 (2008).

[13] N.A. Chernikov and E.A. Tagirov, Ann. Inst. Henri Poincaré 9, 109 (1968); E.A. Tagirov, Ann. Phys. 76, 561 (1973).

[14] P. Candelas and D.J. Raine, Phys. Rev. D 12, 965 (1975).

[15] J.S. Dowker and R. Critchley, Phys. Rev. D 13, 224 (1976); J.S. Dowker and R. Critchley, Phys. Rev. D 13, 3224 (1976).

[16] T.S. Bunch and P.C.W. Davies, Proc. R. Soc. London A 360, 117 (1978).

[17] N.D. Birrell, J. Phys. A 12, 337 (1979).

[18] S.G. Mamayev, Sov. Phys. J. 24, 63 (1981).

[19] A. Vilenkin and L.H. Ford, Phys. Rev. D 26, 1231 (1982).

[20] B. Allen, Nucl. Phys. B 226, 228 (1983); Ann. Phys. 161, 152 (1985).

[21] L.H. Ford, Phys. Rev. D 31, 710 (1985).

[22] K. Kirsten and J. Garriga, Phys. Rev. D 48,567 (1993).

[23] G. Esposito, G. Miele, and L. Rosa, Class. Quantum Grav. 11, 2031 (1994).

[24] T. Prokopec and R.P. Woodart, JHEP 10, 059 (2003); T. Prokopec, O. Tornkvist, and R.P. Woodart, Ann. Phys. 303, 251 (2003); T. Prokopec and R.P. Woodart, Ann. Phys. 312, 1 (2004); T. Prokopec and E. Puchwein, JCAP 04, 007 (2004).

[25] G. Cognola, E. Elizalde, S. Nojiri, S.D. Odintsov, and S. Zerbini, JCAP 02, 010 (2005).

[26] F. Finelli, G. Marozzi, G.P. Vacca, and G. Venturi, Phys. Rev. D 71, 023522 (2005).

[27] A. Dolgov and D.N. Pelliccia, Nucl. Phys. B 734, 208 (2006).

[28] N.D. Birrell and P.C.W. Davies, Quantum Fields in Curved Space (Cambridge University Press, Cambridge, 1982).

[29] A.A. Saharian and M.R. Setare, Phys. Lett. B 659, 367 (2008).

[30] S. Bellucci and A.A. Saharian, Phys. Rev. D 77, 124010 (2008). 
[31] S. Nojiri, S.D. Odintsov, and S. Zerbini, Class. Quantum. Grav. 17, 4855 (2000); W. Naylor and M. Sasaki, Phys. Lett. B 542, 289 (2002); E. Elizalde, S. Nojiri, S.D. Odintsov, and S. Ogushi, Phys. Rev. D 67, 063515 (2003); I.G. Moss, W. Naylor, W. Santiago-German, and M. Sasaki, Phys. Rev. D 67, 125010 (2003); K. Uzawa, Prog. Theor. Phys. 110, 457 (2003); A.A. Saharian and M.R. Setare, Phys. Lett. B 584, 306 (2004); M.R. Setare, Phys. Lett. B 620, 111 (2005); M. Minamitsuji, W. Naylor, and M. Sasaki, Nucl. Phys. B 737, 121 (2006); M.R. Setare, Phys. Lett. B 637, 1 (2006).

[32] A. Flachi, J. Garriga, O. Pujolàs, and T. Tanaka, J. High Energy Phys. 08, 053 (2003); A. Flachi and O. Pujolàs, Phys. Rev. D 68, 025023 (2003); A.A. Saharian, Phys. Rev. D 73, 044012 (2006); A.A. Saharian, Phys. Rev. D 73, 064019 (2006); A.A. Saharian, Phys. Rev. D 74, 124009 (2006).

[33] A.A. Saharian, Class. Quantum Grav., in press, arXiv:08023.2413.

[34] S.M. Christensen, Phys. Rev. D 17, 946 (1978).

[35] P.R. Anderson, W. Eaker, S. Habib, C. Molina-París, and Emil Mottola, Phys. Rev. D 62, 124019 (2000).

[36] A. Pais, J. Math. Phys. 3, 1135 (1962).

[37] C.J. Isham, Proc. R. Soc. London A 362, 383 (1978); C.J. Isham, Proc. R. Soc. London A 364, 591 (1978).

[38] A. A. Saharian, "The Generalized Abel-Plana Formula with Applications to Bessel Functions and Casimir Effect," Report No. IC/2007/082; arXiv:0708.1187.

[39] G. Pérez-Nadal, A. Roura, and E. Verdaguer, Class. Quantum Grav. 25, 154013 (2008).

[40] Th. Kaluza, Sitz. Preuss. Akad. Wiss. K1, 966 (1921); O. Klein, Z. Phys. 37, 895 (1926). 